\title{
A comparison of nontreponemal tests in cerebrospinal fluid for neurosyphilis diagnosis: equivalent detection of specific antibodies
}

\author{
Uma comparação de testes não treponêmicos no líquido cefalorraquidiano para \\ diagnóstico de neurossífilis: detecção equivalente de anticorpos específicos.
}

Isadora Versiani', Mauro Jorge Cabral-Castro², Marzia Puccioni-Sohler ${ }^{1,2}$

\begin{abstract}
Syphilis is a re-emerging sexually-transmitted infection, caused by the spirochete Treponema pallidum, that may penetrate early into the central nervous system. The venereal disease research laboratory test (VDRL) on the cerebrospinal fluid (CSF) is the most widely used for neurosyphilis diagnosis. We evaluated the performance of two other nontreponemal tests (rapid plasma reagin [RPR] and unheated serum reagin [USR] tests) in comparison with the VDRL in CSF. Methods: We analyzed CSF samples from 120 individuals based on VDRL reactivity in the CSF and the clinical picture of neurosyphilis. Results: High inter-rater reliability was found among all three tests, with equivalent sensitivity and specificity. Intraclass correlation coefficient for absolute agreement was 1 for VDRL versus USR, 0.99 for VDRL versus RPR, and 0.99 for RPR versus USR. Conclusions: Rapid plasma reagin and unheated serum reagin tests were identified as excellent alternatives for neurosyphilis diagnosis.
\end{abstract}

Keywords: cerebrospinal fluid; neurosyphilis.

\section{RESUMO}

A sífilis é uma infecção reemergente sexualmente transmissível pelo espiroqueta Treponema pallidum, que pode penetrar precocemente no sistema nervoso central. 0 teste venereal disease research laboratory test (VDRL) no líquido cefalorraquidiano (LCR) é o mais amplamente utilizado para diagnóstico de neurossífilis. Avalia-se o desempenho de dois outros testes não treponêmicos (rapid plasma reagin - RPR and unheated serum reagin - USR tests) em comparação ao VDRL no LCR. Métodos: Foram analisadas amostras de LCR de 120 indivíduos com base no quadro clínico compatível com neurossifilis e reatividade no VDRL no LCR. Resultados: Os testes apresentaram elevada concordância. O coeficiente de correlação intraclasse para concordância absoluta foi de 1 para VDRL versus USR, 0,99 para VDRL versus RPR e 0,99 para RPR versus USR. Conclusões: Os testes rapid plasma reagin e unheated serum reagin foram identificados como excelentes alternativas para o diagnóstico de neurossífilis.

Palavras-chaves: líquido cefalorraquidiano; neurossifilis.

Syphilis is a sexually-transmitted infection caused by the spirochete Treponema pallidum subspecies pallidum ${ }^{1}$. It currently stands out as a re-emerging disease in several regions of the world, with an estimated 10 million new cases per year, with a greater incidence in African and southeast Asian countries. The group of men who have sex with men makes up the majority $(75 \%)$ of affected individuals, half of them also being infected by $\mathrm{HIV}^{2}$. Historically acknowledged as "the great mimicker”, syphilis presents varied clinical features ${ }^{1}$.
The etiological agent penetrates the central nervous system early in $70 \%$ of patients ${ }^{3}$. Central nervous system invasion by Treponema pallidum can lead to a transient or persistent inflammatory disorder. The neurosyphilis may be asymptomatic or progress to meningeal, meningovascular and/or parenchymatous forms ${ }^{2}$.

Laboratory diagnosis is based on treponemal and nontreponemal tests. The former detects specific antibodies against native or recombinant treponemal components, and

${ }^{1}$ Universidade Federal do Estado do Rio de Janeiro, Escola de Medicina e Cirurgia, Rio de Janeiro RJ, Brasil;

${ }^{2}$ Universidade Federal do Rio de Janeiro, Faculdade de Medicina, Pós-Graduação em Doenças Infecciosas e Parasitárias, Rio de Janeiro RJ, Brasil.

Marzia Puccioni-Sohler (iD) https://orcid.org/0000-0001-6007-9142

Correspondence: Marzia Puccioni-Sohler; Rua Mariz e Barros, 775 - Tljuca; 202970-004 Rio de Janeiro RJ, Brasil; E-mail:m_puccioni@yahoo.com.br Conflict of interest: There is no conflict of interest to declare.

Received 21 May 2018; Received in final form 18 September 2018; Accepted 10 October 2018. 
the latter are flocculation tests that detect antibodies against cardiolipin-lecithin-cholesterol complex antigen ${ }^{1,4}$. The nontreponemal tests can be qualitative or quantitative. While the qualitative test indicates the presence or absence of antibody in the sample, the quantitative test determines the titer of antibodies. It is important for monitoring the response to treatment. There are four types of nontreponemal tests based on flocculation methodology: the Venereal Disease Research Laboratory test (VDRL), Unheated Serum Reagin (USR), Rapid Plasma Reagin (RPR) and Toluidine Red Unheated Serum. The VDRL is widely employed in the cerebrospinal fluid (CSF) and presents high specificity (99.13\%) and low sensitivity $(30-70 \%)^{4,5}$. False-negative results are common, which means a negative VDRL cannot exclude the diagnosis. The VDRL and the USR tests require visualization of the result using a microscope. The RPR contains carbon particles that allow the naked eye to read the results, avoiding the use of a microscope ${ }^{6}$.

There is no ideal gold standard test. The lack of an ideal diagnostic test for neurosyphilis causes difficulties in the management of suspected cases, both symptomatic and asymptomatic, delaying treatment and increasing the risk of neurological sequelae. Previous studies of the RPR test show conflicting findings, and there are no studies on the USR test so far. The goal of this research was to analyze nontreponemal RPR and USR tests, in comparison with the VDRL, using CSF from neurosyphilis patients and controls.

\section{METHODS}

\section{Samples and ethics}

We analyzed VDRL-tested CSF specimens, stored at $-20^{\circ} \mathrm{C}$ between 2012 and 2015, from 120 Brazilian patients. Samples were selected and assembled into three groups according to the clinical manifestation of neurosyphilis and the VDRL results in CSF. Group 1: patients with confirmed neurosyphilis (clinical suspicion and reactive VDRL in CSF). Group 2: patients with suspected neurosyphilis (clinical suspicion and nonreactive VDRL in CSF). Group 3: patients without suspected neurosyphilis and nonreactive VDRL in CSF, used as a control. The clinical suspicion of neurosyphilis included the presence of meningeal, vascular or cranial nerve involvement, general paresis, tabes dorsalis or cognitive involvement $^{7}$. For symptomatic patients, neurosyphilis diagnosis is currently based on a clinical picture of neurosyphilis plus a serologic treponemal reactive test, and one of the following results: reactive CSF VDRL, a CSF leukocyte count above 5 cells $/ \mu \mathrm{L}$ or protein levels above $45 \mathrm{mg} / \mathrm{dL}^{8}$. Any CSF samples were excluded from the study if it was taken from patients under 18 years old, the patients suffered a puncture bleeding accident, or those who had not undertaken the VDRL test. This study was approved by the HUCFF/UFRJ ethical committee (CAAE 45939015.1.0000.5257).

\section{Laboratory analysis}

The routine CSF analysis included total and specific cell count by cytosedimentation using the Suta chamber, and determination of protein and glucose concentrations by spectroscopy. Samples were also evaluated for microbiological study - direct testing and culture for common germs, Mycobacterium tuberculosis, fungi and latex for common germs and fungi. Values of cellularity, protein, and glucose were obtained and included in the study.

All nontreponemal tests were performed by two different laboratory professionals, unaware of the VDRL results. All CSF samples were tested by VDRL (WAMA Diagnóstica, São Paulo, Brazil), which was used as the reference test. For qualitative analysis, the USR and RPR tests were adjusted for CSF application using IMMUTREP USR (Omega Diagnostics Ltd, Scotland, UK) and IMMUTREP RPR (Omega Diagnostics LTD, Scotland, UK) following the steps described for serum by the manufacturer. The presence or absence of aggregates (flocculation) was immediately observed under an adequate light source. The absence of flocculation indicates a negative result. All reactive samples were diluted with an isotonic saline solution at $1 / 2$ to $1 / 256$ for semi-quantitative analysis. Positive and negative internal controls were used for all nontreponemal tests used in this study, to validate the results obtained. The CSF samples known to be reactive and nonreactive were used as internal controls.

\section{Statistical analysis}

The SPSS software 20.0 was used for the statistical study. Numerical data were expressed as mean, median and interquartile range (Q1-Q3), and categorical data were expressed as frequency (n) and percentage (\%). In order to compare clinical and laboratory variables among groups, a chi-square test or Fisher exact test was applied for categorical data and Kruskal-Wallis' ANOVA and Dunn multiple comparison tests, for numerical data. The agreement between different CSF tests was analyzed using the intraclass correlation coefficient (ICC) for semi-quantitative measurement. No variables presented a Gaussian distribution, due to normality hypothesis rejection according to the Shapiro-Wilk test ( $\mathrm{p}<0.001$ for all variables). The significance criterion adopted was $5 \%$.

\section{RESULTS}

The demographic characteristics of the 120 participants showed no significant statistical difference $(p>0.05)$ in age or sex, with a slight majority of male patients in all three groups (according to the Dunn comparison test) (Table 1). Group 1 comprised 21 patients diagnosed with neurosyphilis. They exhibited significantly higher levels of leukocytes, monocytes, and neutrophils in CSF, and lower levels of lymphocytes. Group 2, comprising 49 clinically-compromised patients compatible with neurosyphilis, with nonreactive CSF VDRL results, showed total protein significantly lower than Groups 1 and 3, and fewer macrophages (Table 1). 
Table 1. Clinical and laboratorial variable according to study group.

\begin{tabular}{|c|c|c|c|c|c|c|c|c|c|c|}
\hline \multirow{2}{*}{ Variable } & \multicolumn{3}{|c|}{ Group $1(n=21)$} & \multicolumn{3}{|c|}{ Group $2(n=49)$} & \multicolumn{3}{|c|}{ Group $3(n=50)$} & \multirow{2}{*}{$p$ - value ${ }^{1}$} \\
\hline & mean & median & IQR & mean & median & IQR & mean & median & IQR & \\
\hline \multicolumn{11}{|l|}{ Demographic data } \\
\hline Age (years) & 48.9 & 52 & $39-56$ & 48.6 & 46 & $35-57$ & 47.4 & 51 & $33-59$ & 0.88 \\
\hline Male-n (\%) & \multicolumn{3}{|c|}{$15(71.4 \%)$} & \multicolumn{3}{|c|}{$37(75.5 \%)$} & \multicolumn{3}{|c|}{$27(54 \%)$} & 0.066 \\
\hline \multicolumn{11}{|l|}{ CSF cells } \\
\hline Leukocytes (cells/mm³) & 35 & 8 & $4 / 33$ & 6.9 & 2 & $1 / 5$ & 9.4 & 2,5 & $1 / 5$ & $0.0005^{(a)}$ \\
\hline Lymphocytes (\%) & 88 & 89 & $85-100$ & 94 & 100 & $100-100$ & 94 & 100 & $99-100$ & $0.001^{\text {(a) }}$ \\
\hline Monocytes (\%) & 6.4 & 6 & $0-9$ & 2.5 & 0 & $0-0$ & 2.7 & 0 & $0-0.75$ & $0.002^{(a)}$ \\
\hline Neutrophils (\%) & 4.3 & 0 & $0-6$ & 3.5 & 0 & $0-0$ & 3.1 & 0 & $0-0$ & $0.008^{(a)}$ \\
\hline Macrophages (\%) & 1.05 & 0 & $0-1$ & 0 & 0 & $0-0$ & 0.26 & 0 & $0-0$ & $0.0003^{(b)}$ \\
\hline Plasmocytes (\%) & 0.24 & 0 & $0-0$ & 0 & 0 & $0-0$ & 0.14 & 0 & $0-0$ & 0.26 \\
\hline \multicolumn{11}{|c|}{ CSF protein and glucose profile } \\
\hline Protein $(\mathrm{mg} / \mathrm{dL})$ & 116 & 60 & $48-95$ & 50.6 & 41 & $34-57$ & 74.4 & 57 & $39-77$ & $0.002^{(b)}$ \\
\hline Glucose (mg/dL) & 52.2 & 50 & $45-60$ & 58.6 & 56 & $52-64$ & 64.5 & 60 & $49-72$ & 0.083 \\
\hline
\end{tabular}

Group 1: neurosyphilis diagnosis; Group 2: clinical suspicion of neurosyphilis; Group 3: control (without suspicion of neurosyphilis); ${ }^{1}$ Numerical data were expressed by mean, median and interquartile range and compared by Kruskal-Wallis' ANOVA. Categorical data were expressed by frequency ( $\mathrm{n}$ ), percentage (\%) and compared by chi-square test or Fisher exact test. NP: statistical test not performed. According to Dunn's multiple comparison test, at $5 \%$ : (a) group $1 \neq$ groups 2 and 3 and (b) group $2 \neq$ groups 1 and 3 . CSF: cerebrospinal fluid.

Table 2. Results of the semi-quantitative analyzes in the reactive samples of 21 patients suspecting of Neurosyphilis in the group 1 with titers ranging from 2 to 128 by VDRL, RPR and USR tests.

\begin{tabular}{lccc}
\hline \multirow{2}{*}{ Sample } & \multicolumn{3}{c}{ Commercial flocculation kits (Titers) } \\
\cline { 2 - 4 } & VDRL & USR & RPR \\
\hline SYP01 & 4 & 4 & 4 \\
SYP02 & 4 & 4 & 4 \\
SYP03 & 2 & 2 & 2 \\
SYP04 & 16 & 16 & 16 \\
SYP05 & 2 & 2 & 2 \\
SYP06 & 4 & 4 & 4 \\
SYP07 & 4 & 4 & 4 \\
SYP08 & 2 & 2 & 2 \\
SYP09 & 128 & 128 & 128 \\
SYP10 & 4 & 4 & 4 \\
SYP11 & 8 & 8 & 8 \\
SYP12 & 16 & 16 & 16 \\
SYP13 & 4 & 4 & 4 \\
SYP14 & 2 & 2 & 2 \\
SYP15 & 32 & 32 & 16 \\
SYP16 & 4 & 4 & 2 \\
SYP17 & 8 & 8 & 2 \\
SYP18 & 4 & 4 & 2 \\
SYP19 & 4 & 4 & 2 \\
SYP20 & 4 & 4 & 2 \\
SYP21 & 4 & 4 & 2 \\
\hline VPLV & 4 & 4 & 2 \\
\hline
\end{tabular}

VDRL: Venereal Disease Research Laboratory test; USR: Unheated Serum Reagin test; RPR: Rapid Plasma Reagin test.

The VDRL, RPR, and USR were reactive in all 21 patients with a diagnosis of neurosyphilis (Group 1). Reactivity agreement was not analyzed in Groups 2 and 3, since all three tests were negative. The qualitative agreement, a comparison of positive and negative results by kappa values, was not necessary since the VDRL, RPR and
Table 3. Correlation for reactivity among syphilis tests in CSF performed in the group 1 (neurosyphilis).

\begin{tabular}{lcccc} 
Test & $\mathrm{n}$ & $\mathrm{ICC}$ & $95 \% \mathrm{Cl}$ & $\mathrm{p}$-value \\
\hline VDRL versus USR & 21 & 1 & $1-1$ & $<0.0001$ \\
VDRL versus RPR & 21 & 0.99 & $0.97-0.99$ & $<0.0001$ \\
USR versus RPR & 21 & 0.99 & $0.97-0.99$ & $<0.0001$
\end{tabular}

ICC: Intraclass correlation coefficient for absolute agreement; 95\%Cl: $95 \%$ confidence interval for ICC; Note: Two-way mixed model. VDRL: Venereal Disease Research Laboratory test; USR: Unheated Serum Reagin test; RPR: Rapid Plasma Reagin test.

USR tests had equivalent results. All patients in Group 1 were reactive in CSF and all patients in Groups 2 and 3 were not reactive in CSF, for all three tests. The kappa values are therefore equivalent to 1 , indicating perfect qualitative agreement between tests. Using the VDRL as the reference test, the RPR and USR presented a sensitivity and specificity of $100 \%$.

In the semi-quantitative analysis, the titers of the three tests ranged from 2 to 128 in the CSF of 21 patients with neurosyphilis diagnosis (Group 1) (Table 2). The titers were concordant between CSF-VDRL and CSF-USR in 100\%, and between the three tests in 14 out of the 21 reactive samples (66.7\%) (Table 2). Although differences were found in the titration of some samples, the correlation between the tests was statistically significant. Reactivity agreement between RPR, USR and VDRL tests, as a semi-quantitative measurement, was analyzed using the ICC. The closer the ICC is to 1 , the stronger the agreement is, demonstrating quantitative resemblance between compared tests (Table 3). This analysis was performed for Group 1 (neurosyphilis), demonstrating perfect agreement between VDRL and USR (ICC $=1$ ) and excellent agreement between VDRL and RPR (ICC $=0.99$ ) and between RPR and USR in CSF $($ ICC $=0.99)(p<0.0001)$. 


\section{DISCUSSION}

A gold standard diagnostic tool for neurosyphilis does not yet exist. The VDRL test is the most widely-used test due to the high specificity in CSF. We studied two other nontreponemal tests, the RPR and USR tests, as diagnostic alternatives to the VDRL in the CSF of patients with neurosyphilis. We found excellent agreement between all three tests.

Pleocytosis, intrathecal synthesis of total IgG including oligoclonal IgG bands restricted to the CSF may be found in neurosyphilis. These data indicate inflammatory reaction in the CSF and contribute to the demonstration of the possible presence of disease in suspected cases. However, such abnormalities are absent in up to $40 \%$ of patients 9 . According to previous studies, CSF pleocytosis and protein elevation have shown higher specificity (93\%) than sensitivity $(80.8 \%)^{10}$. In our study, the neurosyphilis diagnosis was also associated with cases of CSF abnormalities characterized by an inflammatory profile of elevated cell count and protein concentration.

False negative results occur in nontreponemal tests in the prozone phenomenon, when antibody levels are so high that the test antigen-antibody ratio does not favor flocculation ${ }^{8}$. Positive predictive values may be reduced in the elderly, pregnant women, autoimmune patients and injecting drug users, with a higher number of false positive results ${ }^{8}$. Previous studies of nontreponemal tests show variable findings ${ }^{11,12}$. When the serological RPR test was tested in comparison with the CSF VDRL, an unsatisfactory agreement was observed between the tests ${ }^{13}$. A sensitivity of $40 \%$ and specificity of $85.2 \%$ were found, lower than those of VDRL, which were $50 \%$, and $99.8 \%$, respectively. False-positive RPR results were found for $14 \%$ of their patients, who were suffering from neurological diseases other than neurosyphilis. In contrast, Castro et al..$^{13}$ obtained more satisfactory results for the use of the RPR test in CSF, two decades later. Sensitivity and specificity were estimated at $75 \%$ and $99.3 \%$, both values higher than those found in the CSF VDRL test. In our study, RPR and USR nontreponemal tests were evaluated as diagnostic alternatives to the VDRL test. All three produced equivalent results with excellent agreement. No differences in sensitivity or specificity were found; the three nontreponemal tests that were analyzed presented similar abilities to detect the disease and to give negative results for uninfected patients.

Marra et al. ${ }^{6}$ demonstrated that RPR specificity exceeded that of the CSF VDRL test, which could favor its use as a suitable alternative for diagnosis; however, both tests detected many false negatives, and the RPR test presented even lower sensitivity than the VDRL test. Their study was conducted in immunocompromised HIV-infected patients, and considered non-HIV VDRL values for sensitivity and specificity. In the present study, there were no differences in sensitivity and specificity among both tests, which also favored the possibility of diagnostic alternatives. Zhu et al. detected low sensitivity in the RPR test compared to the VDRL, in spite of satisfactory specificity ${ }^{14}$. The present study found no cases in which VDRL was negative and RPR was positive, whereas Castro et al. ${ }^{13}$ did.

Study limitations should be acknowledged. Due to the use of previously-collected samples and to the laboratory focus of the study, clinical follow-up was not possible. The complete clinical pictures, courses of illness and therapeutic choices of the patients were not known.

Early diagnosis of neurosyphilis is essential for rapid therapeutic implementation, which minimizes neurological sequelae and contributes to a greater understanding of this re-emerging and multifaceted disease. The RPR and USR nontreponemal tests turn out to be excellent alternatives to the CSF VDRL in neurosyphilis diagnosis, presenting similar sensitivity, specificity and material requirements, especially when VDRL is not available, or to confirm VDRL results. It may attend laboratories at various resource levels.

\section{References}

1. Berger J, Dean D. Neurosyphilis. In: Biller J, Ferro J. Handbook of clinical neurology. Amsterdam: Elsevier B.V; 2014. p. 1461-72.

2. Bhai S, Lyons JL. Neurosyphilis update: atypical is the new typical. Curr Infect Dis Rep. 2015 May;17(5):481. https://doi.org/10.1007/s11908-015-0481-x

3. Ho EL, Lukehart SA. Syphilis: using modern approaches to understand an old disease.J Clin Invest. 2011 Dec;121(12):4584-92. https://doi.org/10.1172/JCl57173

4. Ministério da Saúde (BR), Secretaria de Vigilância em Saúde, Departamento de Vigilância, Prevenção e Controle das Doenças Sexualmente Transmissíveis, AIDS e Hepatites Virais. Manual técnico para diagnóstico da sífilis. Brasília, DF: Ministério da Saúde; 2016.

5. Golden MR, Marra CM, Holmes KK. Update on syphilis: resurgence of an old problem. JAMA. 2003 Sep;290(11):1510-4. https://doi. org/10.1001/jama.290.11.1510

6. Marra CM, Tantalo LC, Maxwell CL, Ho EL, Sahi SK, Jones $T$. The rapid plasma reagin test cannot replace the venereal disease research laboratory test for neurosyphilis diagnosis. Sex Transm Dis. 2012 Jun;39(6):453-7. https://doi.org/10.1097/OLQ.0b013e31824b1cde

7. Centers for Disease Control and Prevention (US), Department of Health and Human Services. Syphilis: a provider's guide to treatment and prevention. Atlanta: Centers for Disease Control and Prevention; 2017.

8. Marra CM. Neurosyphilis. Continuum (Minneap Minn). 2015 Dec;21 6 Neuroinfectious Disease:1714-28

9. Zhang HL, Lin LR, Liu GL, Zeng YL, Wu JY, Zheng WH, et al. Clinical spectrum of neurosyphilis among HIV-negative patients in the modern era. Dermatology. 2013;226(2):148-56. https://doi.org/10.1159/000347109

10. Lin LR, Lin DH, Tong ML, Liu LL, Fan JY, Zhu XZ, et al. Macrophage migration inhibitory factor as a novel cerebrospinal fluid marker for neurosyphilis among HIV-negative patients. Clin Chim Acta. 2016 Dec;463:103-8. https://doi.org/10.1016/j.cca.2016.10.018 
11. Ho EL, Marra CM. Treponemal tests for neurosyphilis: less accurate than what we thought? Sex Transm Dis. 2012 Apr;39(4):298-9. https://doi.org/10.1097/OLQ.0b013e31824ee574

12. Tong ML, Zhang HL, Zhu XZ, Fan JY, Gao K, Lin LR, et al. Re-evaluating the sensitivity of the rabbit infectivity test for Treponema pallidum in modern era. Clin Chim Acta. 2017 Jan;464:136-41. https://doi. org/10.1016/j.cca.2016.11.031

13. Castro R, Prieto ES, Pereira FLM. Nontreponemal tests in the diagnosis of neurosyphilis: an evaluation of the Venereal Disease
Research Laboratory (VDRL) and the Rapid Plasma Reagin (RPR) tests. J Clin Lab Anal. 2008;22(4):257-61. https://doi.org/10.1002/ jcla. 20254

14. Zhu L, Gu X, Peng RR, Wang C, Gao Z, Zhou P, et al. Comparison of the cerebrospinal fluid (CSF) toluidine red unheated serum test and the CSF rapid plasma reagin test with the CSF venereal disease research laboratory test for diagnosis of neurosyphilis among HIV-negative syphilis patients in China.J Clin Microbiol. 2014 Mar;52(3):736-40. https://doi.org/10.1128/JCM.02522-13 